\title{
Space-Time Intervals Underlie Human Conscious Experience, Gravity, and a Theory of Everything
}

\author{
Richard Sieb
}

\begin{abstract}
Space-time intervals are the fundamental components of conscious experience, gravity, and a Theory of Everything. Space-time intervals are relationships that arise naturally between events. They have a general covariance (independence of coordinate systems, scale invariance), a physical constancy, that encompasses all frames of reference. There are three basic types of space-time intervals (light-like, time-like, space-like) which interact to create space-time and its properties. Human conscious experience is a four-dimensional space-time continuum created through the processing of space-time intervals by the brain; space-time intervals are the source of conscious experience (observed physical reality). Human conscious experience is modeled by Einstein's special theory of relativity, a theory designed specifically from the general covariance of space-time intervals (for inertial frames of reference). General relativity is our most accurate description of gravity. In general relativity, the general covariance of space-time intervals is extended to all frames of reference (inertial and non-inertial), including gravitational reference frames; space-time intervals are the source of gravity in general relativity. The general covariance of space-time intervals is further extended to quantum mechanics; space-time intervals are the source of quantum gravity. The general covariance of space-time intervals seamlessly merges general relativity with quantum field theory (the two grand theories of the universe). Space-time intervals consequently are the basis of a Theory of Everything (a single all-encompassing coherent theoretical framework of physics that fully explains and links together all physical aspects of the universe). This theoretical framework encompasses our observed physical reality (conscious experience) as well; space-time intervals link observed physical reality to actual physical reality. This provides an accurate and reliable match between observed physical reality and the physical universe by which we can carry on our activity. The Minkowski metric, which defines generally covariant space-time intervals, may be considered an axiom (premise, postulate) for the Theory of Everything.
\end{abstract}

Key Words: experience; relativity; quantum; gravity; space-time intervals; general covariance

\section{Introduction}

Gravity or gravitation is a natural phenomenon in which all things with mass are brought toward (gravitate toward) one another. For most applications, gravity is well approximated by Newton's Law of Universal Gravitation, which describes gravity as a force causing any two bodies to be attracted to each other, with a force proportional to the product of their masses and inversely proportional to the square of the distance between them. Gravity, however, is most accurately described by the general theory of relativity (Einstein, 2001), which describes gravity as the curvature of space-time caused by the local distribution of mass, momentum, and energy. General relativity is a geometric theory of gravitation in which gravity is described, not as a force, but as the structure (geodesics) of space-time. Quantum mechanics 
seeks to describe gravity (quantum gravity) according to the principles of quantum field theory, as a force. Consequently, quantum mechanics is incompatible with general relativity. However, if quantum gravity is also described, not as a force, but as the structure of space-time, then quantum mechanics seamlessly merges with general relativity, providing a unified theory of gravity. Such a theory would unify the fundamental forces of nature producing a Theory of Everything (a single all-encompassing coherent theoretic framework of physics which fully explains and links together all physical aspects of the universe-Weinberg, 1993).

The notion of space-time is fundamental to the understanding of gravity. Space may be defined as the boundless three-dimensional extent in which events have relative position and direction. Time is a continuum in which events succeed one another from past, through present, to future. Space is often conceived in three linear dimensions (length, width, height), but modern physicists usually consider it with time, as part of a boundless four-dimensional continuum called space-time. Space-time is any mathematical model that combines space and time into a single continuum (Petkov, 2010). Notice that space and time are both defined in relation to events. In physics, particularly relativity, an event is a point in space-time (a specific place and time) and the physical situation or occurrence associated with it (French, 1968). The notion of an event is an idealization, in the sense that it specifies a definite time and place, whereas any actual event has a finite extent, both in time and place (Sartori, 1996). Space and time have little meaning and structure for a single isolated event. However, as soon as more than one event occurs, space and time acquire meaning and structure; relationships arise naturally between events and it is these relationships which give space and time meaning and structure (creating space-time). There are a number of relationships that arise naturally between events: separation in space, separation in time, separation in space-time (space-time interval), cause-effect, past-future. These relationships are all that are needed for the creation of space-time and its properties.

Space-time has had a different meaning in general relativity and quantum mechanics. Quantum mechanics has depended since its inception on a fixed space-time background structure. In quantum mechanics, space-time is a pre-existing arena in which events happen; quantum theory merely gives the rules for these happenings (Smolin, 2001). A fundamental lesson of general relativity is that there is no fixed space-time background, space-time is dynamic (Einstein, 2001; Smolin, 2001). Space and time arise out of the relationships between events; space-time has no existence apart from the events that occur within it (Smolin, 2001). While easy to grasp in principle, this is the hardest idea to understand about general relativity, and its consequences are profound and not fully explored, even at the classical level. To a certain extent, general relativity is a relational theory (Feynman et al., 1995), in which the only physically relevant information is the relationships between events (Smolin, 2001). It is this dynamic notion of space-time that facilitates the description of gravity. If the dynamic notion of space-time is extended to quantum mechanics, then a quantum theory of gravity may be described, which seamlessly merges quantum mechanics with general relativity.

Space-time, the conventional thinking goes, is not made up of anything at all. Scientists, however, have begun to question this convention. Space-time may actually be composed of tiny chunks of information which interact to create space-time and its properties (Moskowitz, 2017). This notion, if correct, would not just explain space-time, it could also help achieve a long-sought quantum theory of gravity, which would merge general relativity and quantum mechanics, the two grand theories of the universe (Moskowitz, 2017). This prognosticates space-time intervals. Space-time intervals are universal relationships which arise naturally between events. As will be seen, space-time intervals are tiny chunks of 
information (what, when, where) which interact to create space-time and its properties. Spacetime intervals not only explain space-time, they also underlie a quantum theory of gravity, which merges general relativity and quantum mechanics, providing a Theory of Everything.

\section{Space-time Intervals}

An event is the fundamental entity of observed physical reality represented by three coordinates of space and one coordinate of time in the space-time continuum postulated by the theory of relativity. In such a four-dimensional frame of reference (an observational perspective of space described using a coordinate system), one can assign four coordinates to an event: three spatial coordinates $(x, y, z)$ to describe the location of the event and a time coordinate $(t)$ to specify the moment at which the event occurs (Petkov, 2010). An event has a unique position in space-time specified by four coordinates $(x, y, z, t)$. In relativity, the unification of space and time is exemplified by the common practice of selecting a metric (a system or standard of measurement of distance) such that all four dimensions are measured in terms of units of distance $(x, y, z, c t)$, where " $c$ " represents the speed of light in a vacuum and "ct" represents distance along the time axis (Petkov, 2010). $t$ is the time (in seconds) light travels at $c$ (at $186,000 \mathrm{miles} / \mathrm{sec}$ or $300,000 \mathrm{~km} / \mathrm{sec}$ ); hence "ct" represents the distance light travels and consequently distance along the time axis. Since $c$ is a universal physical constant equal to 1 (unity), ct reduces to $t$. This enables mathematical description of space-time, the positions of events in space-time, and most importantly, space-time intervals.

In our three-dimensional Euclidean space, the shortest distance between two points is known as the regular Euclidean distance (this distance is purely spatial and always positive). The regular Euclidean distance is given by the Pythagorean Theorem: the square of the hypotenuse (the regular Euclidean distance) of a right triangle is equal to the sum of the squares of the other two sides. The metric of a (mathematical) space defines distance in that space. Hence the Euclidean metric is the Pythagorean Theorem; it defines distance in 3D Euclidean space. The regular Euclidean distance is preserved (invariant) in all Euclidean inertial (constant velocity) frames of reference (by the Euclidean transformations), i.e., the regular Euclidean distance is independent of the coordinate system (it is scale invariant) for Euclidean inertial frames. This is known as general covariance. A physical law expressed in a generally covariant fashion (like the Euclidean metric) takes the same mathematical form in all coordinate systems (it has a physical constancy) and is usually expressed in terms of tensor fields (O'Hanian and Ruffini, 1994). Tensors are mathematical objects which describe relationships; this makes them inherently generally covariant and therefore useful in representing general covariance. In Newtonian physics, the universe is assumed to be totally Euclidean, and we use the $3 \mathrm{D}$ Euclidean metric to define all of our distances. When time is taken as a fourth dimension, the Galilean transformations preserve the $3 \mathrm{D}$ Euclidean distance and time separately in all Euclidean inertial frames. Einstein realized that 3D Euclidean geometry was insufficient to describe electromagnetic effects and the constancy (general covariance) of $c$; Einstein consequently developed his special theory of relativity in 1905 to explain these effects (Einstein, 2001). The mathematical structure of Minkowski space-time was an immediate consequence of the postulates of special relativity (Landau and Lifshitz, 1960; Petkov, 2010). In Minkowski space-time, space and time are interwoven. The shortest distance between two points (events) in Minkowski space-time is given by the invariant space-time interval between the events, which takes into account not only their spatial separation, but their temporal separation as well (Petkov, 2010). Minkowski space-time is a combination of 3 -dimensional Euclidean space and time into a 4-dimensional manifold (coordinate system) where the space-time interval between any two events is independent of the inertial frame of reference in which it was recorded, i.e., space-time intervals are generally covariant (constant) for all Minkowski inertial frames. In 
Minkowski space-time, the invariant space-time interval replaces the invariant regular Euclidean distance of Euclidean space.

The Euclidean metric (Pythagorean Theorem) defines the invariant regular Euclidean distance in Euclidean inertial frames. The Minkowski metric defines the invariant space-time interval in Minkowski inertial frames. The Minkowski metric is defined as to yield the invariant space-time interval between two events when given their coordinate difference vectors (Petkov, 2010; Schutz, 2004):

(space-time interval) " $\mathrm{s}^{2}$ " is equal to the difference between the space coordinates " $\Delta r^{2}$ " of two events minus the difference between the time coordinates " $c^{2} \Delta t^{2}$ " of the two events

(space-time interval) $s^{2}=\Delta r^{2}-c^{2} \Delta t^{2}$ or $s^{2}=\Delta r^{2}-\Delta t^{2}$, if $c=1$

This is the Minkowski metric; it defines distance in Minkowski space-time (the invariant spacetime interval). The Minkowski metric is a physical law expressed in a generally covariant fashion; it has the same form in all Minkowski inertial frames (it has a physical constancy). In practice, the square is utilized, as the metric is a generalization of the Euclidean metric (Pythagorean Theorem) to four-dimensional Minkowski space-time. $r$ is a displacement vector equal to $x+y+z$. Equipped with this metric, the mathematical model of space-time is called Minkowski space-time. Minkowski space-time is a specific example of a Lorentzian manifold (coordinate system); in fact, Minkowski space-time is the simplest example of a Lorentzian manifold (Chen, 2011; Misner et al., 1973; O’Neill, 1983; Petkov, 2010; Ratcliffe, 2006). In a standard Lorentzian manifold, one coordinate is written with a sign (-) opposite to the sign (++ $+\ldots)$ of the other coordinates; this convention allows quantities to be categorized as light-like, time-like, or space-like (Chen, 2011; Misner et al., 1973; O’Neill, 1983; Ratcliffe, 2006). For the Minkowski metric, $t$ is written as negative because it is an imaginary dimension. For the Minkowski metric, the space-time interval " $s$ " is zero, positive, or negative; this allows the definition of three possible types of space-time intervals. When $s^{2}$ is zero, the metric is lightlike; when $s^{2}$ is negative, the metric is time-like; when $s^{2}$ is positive, the metric is space-like. The metric allows not only the definition, classification, and computation of invariant spacetime intervals, it also defines the geometry and properties of Minkowski space-time.

Minkowski space-time is the model of space-time for special relativity, quantum mechanics, and human conscious experience (Chen, 2011; O'Neill, 1983; Sieb, 2015, 2016, 2017a, 2017b). The 4D Lorentzian manifold is also of great importance for general relativity; flat Minkowski space-time provides a springboard for general relativity, as curved space-time is locally Minkowski (Chen, 2011; O’Neill, 1983). It can be seen therefore that the general covariance of space-time intervals (as defined by the Minkowski metric) encompasses special relativity, quantum mechanics, human conscious experience, and general relativity; it encompasses everything.

\section{The Light Cone}

Space-time intervals are also defined by light. A pulse of light emitted from a point spreads out in all directions at $c$. If light is confined to a two-dimensional plane, the light from a flash spreads out in a circle (Figures 1 and 2). If the growing circle is drawn with the vertical axis representing time (Figures 1 and 2), the result is a light cone (Penrose, 2005; Petkov, 2010). In reality, there are three space dimensions, so light would actually form an expanding sphere and the light cone would be a $4 \mathrm{D}$ version of a cone. The concepts are easier to visualize with the number of space dimensions reduced to two. A light cone represents the path that a flash of 
Figure 1: Space-time Intervals. From Wikipedia, 2017
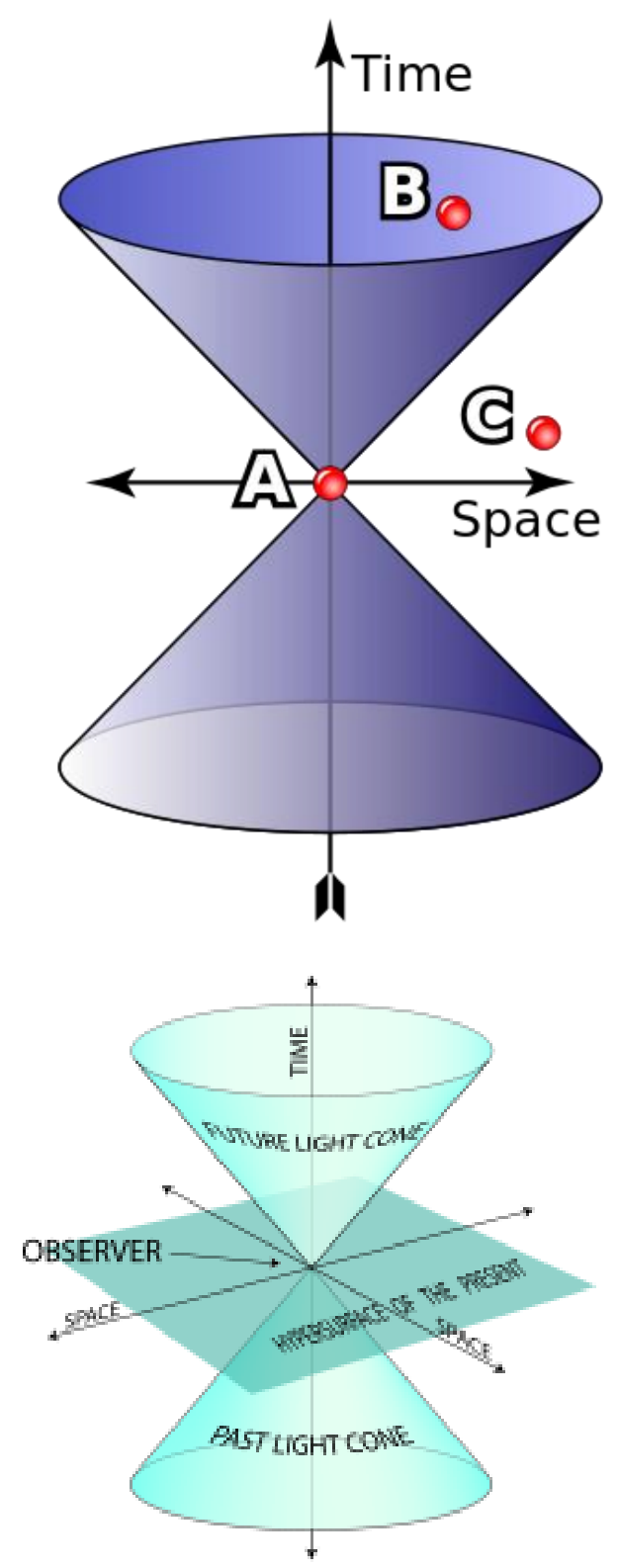


\section{Figure 2: Past and Future Light Cones. From Wikipedia, 2017}

light, emanating from a single event and travelling at the speed of light in all directions, would take through space-time. Light cones are the same for all events and for all observers, since $c$ is a universal physical constant. Given an event A, all events that can be reached by a light pulse from A form a future light cone, while all events that can send a light pulse to A form an inverted past light cone (Figures 1 and 2). Three basic types of space-time intervals are defined by the light cone and the Minkowski metric and play a crucial role in the creation of space-time.

Space-time intervals and $c$ are both generally covariant (they are both scale invariant, independent of coordinate systems, have a physical constancy). Both achieve their general covariance (physical constancy) in the same way; in fact, the general covariance of space-time intervals was developed (special relativity) to explain the physical constancy (general covariance) of $c$. Electromagnetic radiation (includes light) is described as a self-propagating transverse oscillating wave of electric and magnetic fields that are always in phase and at 90 degrees to each other (Brown, 2013; Purcell and Morin, 2013). A time change in one type of field is always matched by a space change in the other, thus maintaining the constancy of $c$. Similarly, for space-time intervals, a time change is also always matched by a space change, thus maintaining the constancy of space-time intervals. The space changes and time changes occur without either causing the other (Einstein, 2001; Purcell and Morin, 2013).

\section{Light-Like Space-time Intervals}

For the Minkowski metric $\left(s^{2}=\Delta r^{2}-c^{2} \Delta t^{2}\right)$, when the difference in the space coordinates of two events is exactly equal to the difference in the time coordinates of the events $\left(\Delta r^{2}=c^{2} \Delta t^{2}\right)$, the space-time interval is zero $\left(s^{2}=0\right)$, the events fall on a light-cone, and the distance between the two events is called a light-like or "null" space-time interval. The spatial distance between the events $\left(\Delta r^{2}\right)$ is equal to the distance light travels $\left(c^{2} \Delta t^{2}\right)$; the two events are separated at $c$. Events occurring to a photon, as it travels along its path, and events on a light cone (see Figures 1 and 2) are of this nature; this occurs when you directly observe a light source or an event (the event is visible because of reflected light). Electromagnetic radiation (including light) carries electromagnetic energy and momentum (Misner et al., 1973; Purcell and Morin, 2013) and may be called the electromagnetic interaction; signals or information can be passed between events at $c$ by the electromagnetic interaction. Light-like space-time intervals carry the electromagnetic interaction (light-like space-time separation is necessary for the electromagnetic interaction to occur). The electromagnetic interaction (light-like space-time intervals) helps determine the nature of conscious events through the conveyance of defining information about the events to the eyes ("what" information); this is an electromagnetic (lightlike) transfer of information (Sieb, 2015, 2016, 2017a, 2017b). The electromagnetic interaction (light-like space-time intervals) may be considered a fundamental component of human conscious experience. Since human conscious experience is a space-time continuum (Sieb, 2015, 2016, 2017a, 2017b), the electromagnetic interaction (light-like space-time intervals) is a fundamental interaction involved in the creation of the space-time and properties of human conscious experience. Since energy and mass are equivalent (special relativity), all forms of energy, including light, cause gravitation and are under the influence of it (Einstein, 2001). Hence the electromagnetic interaction (light-like space-time intervals) contributes to gravitation. There is an electromagnetic force field acting on matter which contributes to gravitation; this force field is described by the electromagnetic energy-momentum tensor (Misner et al., 1973; Purcell and Morin, 2013). The electromagnetic interaction is responsible for practically all the phenomena encountered in daily life above the nuclear scale (Ulaby, 2010). It is also an accepted fundamental interaction in quantum mechanics (Ulaby, 2010). All the forces involved in interactions between atoms can be explained by the electromagnetic interaction acting between the electrically charged atomic nuclei and the electrons of the atoms. 
The electromagnetic interaction explains how particles carry momentum, the forces involved in pushing or pulling ordinary material objects, and all sorts of chemical and electrical phenomena (Ulaby, 2010). The electromagnetic interaction is carried by light-like space-time intervals; light-like space-time intervals are the electromagnetic interaction. The electromagnetic interaction (light-like space-time intervals) may be considered a fundamental interaction involved in gravitation and the creation of space-time.

\section{Time-Like Space-time Intervals}

For the Minkowski metric $\left(s^{2}=\Delta r^{2}-c^{2} \Delta t^{2}\right)$, if the difference in the time coordinates of two events is greater than the difference in the space coordinates of the events $\left(c^{2} \Delta t^{2}>\Delta r^{2}\right)$, the space-time interval is negative $\left(s^{2}<0\right)$, the events fall inside a light cone (A and B in Figure 1), and the distance between the events is called a time-like space-time interval. For a time-like space-time interval, the spatial distance between the events is less than the distance light travels; hence there is enough time between the occurrence of the events that signals or information can pass between the events at less than the speed of light. A reference frame exists where two events may be separated only by time, but not by space. The events may occur at the same spatial position, but not at the same time. The events may occur at the same spatial position, but at different times (the events are separated only by time). Time-like space-time intervals account for the experience of conscious events at the same spatial positions, but at different times (this can occur only with time-like separation of the events). Time-like spacetime intervals appear to be a fundamental component of human conscious experience (Sieb, $2015,2016,2017 \mathrm{a}, 2017 \mathrm{~b})$. Since human conscious experience is a space-time continuum, timelike space-time intervals are a fundamental component in the creation of the space-time of human conscious experience. Because signals and other causal influences cannot travel faster than the speed of light, time-like space-time intervals define the concept of causality (Einstein, 2001; Misner et al., 1973). Since for time-like space-time intervals (inside light cones), signals or information can travel between the events at less than the speed of light, one event can influence or be influenced by the other event, by signals or information that does not need to travel faster than the speed of light. One event could be the cause or effect of the other event. If $\mathrm{B}$ causes A, B exists in the past history (in the past light cone) of A (Figures 1 and 2). If $\mathrm{B}$ is caused by A, B exists in the future (in the future light cone) of A (Figures 1 and 2). The past light cone of an event represents the boundary of its causal past and the future light cone the boundary of its causal future. Events with time-like space-time interval separation may be said to have a past or future relation. This can be observed in ordinary human conscious experience. Conscious events which occur at the same position in space, but at different times (time-like space-time interval separation), often have a cause-effect relationship and necessarily a pastfuture relationship (Sieb, 2015, 2016, 2017a, 2017b). Time-like space-time intervals carry the "when" information for the creation of human conscious experience. Time-like space-time interval separation allows signals or information to pass between events, a stress-pressure interaction (stress is the physical pressure, pull, or force exerted by one thing on another; pressure is the physical force exerted on or against an object by something in contact with it). Time-like space-time intervals may be considered a stress-pressure interaction (time-like spacetime interval separation is necessary for the stress-pressure interaction to occur). This stresspressure interaction contributes to gravitation. There is a pressure force field acting on matter, which contributes to gravitation; this force field is described by the pressure energy-momentum tensor (Misner et al., 1973). Time-like space-time intervals may be considered a stress-pressure interaction that participates in gravitation. The strong nuclear fundamental interaction of quantum field theory is a stress-pressure interaction; it is involved in the binding of quarks together to form hadrons (protons and neutrons), and the latter together to form atomic nuclei (Ulaby, 2010). The stress-pressure interaction is carried by time-like space-time intervals; timelike space-time intervals are the stress-pressure interaction. The stress-pressure interaction 
(time-like space-time intervals) may be considered a fundamental interaction for gravitation and the creation of space-time.

\section{Space-Like Space-time Intervals}

For the Minkowski metric $\left(s^{2}=\Delta r^{2}-c^{2} \Delta t^{2}\right)$, if the difference in the space coordinates of two events is greater than the difference in the time coordinates of the events $\left(\Delta r^{2}>c^{2} \Delta t^{2}\right)$, the space-time interval is positive $\left(s^{2}>0\right)$, the events fall outside a light cone (A and C in Figure 1), and the distance between the events is called a space-like space-time interval. For a space-like space-time interval, the spatial distance between the events is greater than the distance light travels and therefore there is not enough time between the occurrence of the events that signals or information can travel between the events at less than the speed of light. One event cannot therefore be the cause or effect of the other event. There exists a reference frame where two events may be separated only by space, but not by time. The events may occur at the same time, but not at the same spatial position. The events may occur simultaneously, but at different spatial positions (the events are separated only by space). Space-like space-time intervals could account for the experience of conscious events simultaneously, but at different spatial positions (this can occur only with space-like separation of the events). Space-like space-time intervals appear to be a fundamental component of human conscious experience (Sieb, 2015, 2016, $2017 \mathrm{a}, 2017 \mathrm{~b})$. Since human conscious experience is a space-time continuum, space-like spacetime intervals are a fundamental interaction in the creation of the space-time of human conscious experience. Generally, the events are considered not to have a cause-effect or pastfuture relation. This can be observed in ordinary conscious experience. Conscious events which occur simultaneously, but in different spatial positions (space-like separation of events), do not have cause-effect or past-future relationships. Space-like space-time intervals carry the "where" information for the creation of human conscious experience. Space-like space-time intervals are a dissipation interaction, an interaction that dissipates or distributes events in space (Misner et al., 1973). This dissipation interaction contributes to gravitation. There is a dissipation force field acting on matter, which contributes to gravitation; this force field is described by the dissipation energy-momentum tensor (Misner et al., 1973). The weak nuclear fundamental interaction of quantum field theory is a dissipation interaction; it acts on the nucleus and mediates radioactive decay (Ulaby, 2010). The dissipation interaction is carried by space-like space-time intervals (space-like space-time separation is necessary for the dissipation interaction to occur); space-like space-time intervals are the dissipation interaction. The dissipation interaction (space-like space-time intervals) may be considered a fundamental interaction for gravitation and the creation of space-time.

\section{Human Conscious Experience}

Space-time intervals account for human conscious experience (Sieb, 2015, 2016, 2017a, 2017b). If one examines any conscious experience, many of the conscious events occur simultaneously in different places (space-like space-time interval separation); other conscious events occur at the same place, but at different times (time-like space-time interval separation); while all conscious events have their qualities or properties (qualia) defined by light (light-like space-time interval separation). Human conscious experience appears to be a space-time continuum created from the processing of the three basic types of space-time intervals by the brain (Sieb, 2015, 2016, 2017a, 2017b). In effect, the human brain utilizes space-time intervals to link (associate) conscious events to form coherent four-dimensional conscious experiences. Space-time intervals explain why conscious experience appears to us the way it does, i.e., the subjectivity of human conscious experience. Conscious experience is essentially an orientation in space and time (an awareness of the existing situation with reference to space, time, and identity); it is an understanding of the position of the observer in space and time (space-time). Conscious experience is our observed physical reality. It allows us to observe, interpret, and 
understand our environment (perception) and to rapidly compose immediate and successful voluntary intentional goal-directed adaptive responses (Sieb, 2004, 2007, 2011, 2013). Consciousness is essential for this function and has made human beings very successful.

A neural correlate for conscious experience has been found in the human brain (Sieb, 2015, 2016, 2017a, 2017b). Light-like space-time interval processing by the brain carries electromagnetic information for perception of the qualities or properties (qualia) of conscious events ("what" information), a fundamental component of human conscious experience (the electromagnetic fundamental interaction). Time-like space-time interval processing by the brain carries information about the flow of conscious events in time and allows the establishment of cause-effect and past-future relationships between conscious events ("when" information), another fundamental component of human conscious experience (the stresspressure fundamental interaction). Space-like space-time interval processing by the brain carries information about the dissipation or distribution of conscious events in space ("where" information), a third fundamental component of human conscious experience (the dissipation fundamental interaction). Space-like and time-like space-time interval processing defines the context of conscious events, i.e., the setting by which conscious events can be fully assessed, understood, and responded to (Sieb, 2015, 2016, 2017a, 2017b). Space-time intervals are the basis for higher cognitive processing: attention, perception, learning, memory, logic, reasoning, planning, calculating, decision-making, problem-solving, and the creation of voluntary intentional goal-directed adaptive actions (Sieb, 2015, 2016, 2017a, 2017b). The three basic types of space-time intervals appear to be the fundamental interactions involved in the creation of human conscious experience, i.e., in the creation of the space-time of human conscious experience. Space-time intervals may be considered tiny chunks of information which are extracted from physical reality to create the dynamic space-time of human conscious experience. In essence, it is the occurrence of the events themselves which creates conscious experience of the events.

Human conscious experiences are self-generated (subjective) internal explicit states, four-dimensional, nonlinear (are more than the linear combination of their input), emerge rapidly in recurrent systems, take time to form, have a prolonged duration, are seamless, structurally-complex, ineffable, transparent, bounded, unified and coherent, informative, serial, limited in capacity, subject to interference, new or novel, variable, flexible, project outwards, can gain access to other systems, arise from attention, and are based on three core sources of information: what, when, and where (Baars, 1988; Koch, 1998; Sieb, 2004, 2007, 2011, 2013, 2015, 2016; 2017a, 2017b; Tononi and Edelman, 1998). These properties of human conscious experience arise from the processing of the three basic types of space-time intervals by the brain.

The creation of the space-time of human conscious experience by the processing of space-time intervals by the brain tells us a number of things. Human conscious experience is a four-dimensional Lorentzian manifold called Minkowski space-time and the Minkowski metric defines its construction. Three basic types of space-time intervals are all that is needed for the construction of human conscious experience, i.e., space-time intervals are the fundamental components of human conscious experience. The three basic types of space-time intervals carry three core types of information (what, when, where) that are utilized in the construction of human conscious experience, i.e., human conscious experience (and basically all human conscious activity) is based on the processing of what, when, and where information carried by space-time intervals (Sieb, 2017a). The processing of the three basic types of space-time intervals by the brain makes possible all cognitive processing, i.e., all higher brain function. All conscious experiences evolve from the processing of the three basic types of space-time intervals by the brain. Everything we know, have known, and will come to know (all observed physical phenomena) comes to us through conscious experience and therefore arises from the processing 
of the three basic types of space-time intervals. The human brain appears to have evolved the ability to create a four-dimensional space-time continuum called conscious experience from the processing of three basic types of space-time intervals. This is our observed physical reality. One has therefore, a single all-encompassing coherent theoretical framework of physics which fully explains and links together all observed physical phenomena and the Minkowski metric is the axiom (premise, postulate) for this framework. Since the brain has selected space-time intervals as the natural basis of its conscious processing, perhaps the three basic types of spacetime intervals are more fundamental than imagined. They may be the fundamental basis of, not just of all observed physical phenomena, but of all physical aspects of the universe (everything). This is interesting because space-time intervals could then link or match our observed physical reality with actual physical reality so that we might thereby extract accurate reliable information from physical reality to determine our activity.

\section{Einstein's Special Theory of Relativity}

It was during my study of Einstein's special theory of relativity that I first noticed that space-time intervals account for the organization of human conscious experience; special relativity actually models human conscious experience (Sieb, 2015, 2016, 2017a, 2017b). Special relativity is based on two postulates: (1) the laws of physics are invariant (the same) in all inertial (constant velocity) frames of reference, and (2) the speed of light (c) is the same for all observers, regardless of the motion of the light source (Einstein, 2001). General covariance is the heart of both postulates; the general covariance of space-time intervals is the basis for special relativity. In special relativity, time cannot be separated from space, because the observed rate at which time passes depends on the relative velocity of the observer. Time and space are interwoven into a single continuum called Minkowski space-time.

Frames of reference can be divided into two groups: inertial (constant velocity) and non-inertial (accelerating). An inertial frame of reference moves with constant velocity in a straight line (Fields, 2015). The physics of an inertial frame has no causes external to the system (Ferraro, 2007). All inertial frames are in a state of constant rectilinear motion with respect to one another, there is zero acceleration. If an acceleration or extraneous force does occur, then the inertial frame is deflected (curved) from its rectilinear trajectory (it is then a non-inertial frame). Measurements in one inertial frame can be converted to measurements in another inertial frame by a simple transformation (the Lorentz transformation in special relativityEinstein, 2001; Guilini, 2005). The Lorentz transformation is a coordinate transformation between two Minkowski reference frames that are moving at constant velocity relative to one another. The transformation connects the space and time coordinates of an event as measured by an observer (someone who takes measurements) in each inertial frame. In all inertial frames (regardless of their relative velocity), the difference between the space coordinates of any two events minus the difference between the time coordinates of the events (the space-time interval) is always the same (invariant). This general covariance is the defining property of the Lorentz transformation. The Lorentz transformation has a number of unintuitive features: observers moving at different inertial velocities may measure different distances, elapsed times, and even different orderings of events, but always such that the speed of light and space-time intervals are invariant in the inertial frames. Special relativity has the following consequences, which have been validated experimentally: length contraction (shortening) and time dilation (slowing) for increased inertial velocity, space-time interval invariance (time dilates and lengths contract at higher speeds of a reference frame relative to another to keep space-time intervals and $c$ invariant in the inertial frames), mass-energy equivalence $\left(E=m c^{2}\right)$, relativistic mass (mass increases with velocity because of increased kinetic energy), a universal speed limit (c), and relativity of simultaneity (Disalle, 2009; Einstein, 2001; Feynman, 1998; Roberts and Schleif, 
2007). The theory is "special" because it refers to the special case of inertial motion, where gravity is negligible.

It is not surprising that special relativity models human conscious experience. Einstein utilized his own conscious experiences (imagination, thought experiments, diagrams,

descriptions, discussions) for the development of his special theory (Hossenfelder, 2015). Einstein in effect analyzed his own conscious experiences to come up with the principles of special relativity. What Einstein actually discovered in his special theory was a model of human conscious experience. Einstein was attempting to describe and explain observed physical reality. One might say that Einstein was successful in this endeavor, as our conscious experience is our observed physical reality.

\section{Einstein's General Theory of Relativity}

Our current understanding of gravity is based on Einstein's general theory of relativity (Einstein, 2001), which is formulated within the framework of classical physics (classical physics refers to all physical theories which are not based on the quantization paradigm-Morin, 2008). There was an inherent limitation to special relativity: the principle of relativity (the general covariance of space-time intervals) could only apply to inertial frames of reference, where gravity is negligible. Consequently, Einstein developed his general theory of relativity to provide a unified theory for both inertial (constant velocity) and non-inertial (accelerated) reference frames (which includes gravitational reference frames). Einstein proposed the principle of general covariance for his special theory of relativity; however, Einstein recognized that the general principle of relativity should also apply to accelerated relative motions (including gravitational), and he used the tool of tensor calculus to extend the special theory's general covariance to all frames, eventually producing the general theory of relativity.

The starting point of general relativity is the Equivalence Principle or the Principle of Universality of Free Fall. The weak equivalence principle states that free-falling bodies (influenced only by gravity) at the same initial conditions (position, velocity) have the same acceleration independent of size and composition (Wessen, 2006). The Einsteinian equivalence principle states that the outcome of any non-gravitational experiment in a freely-falling laboratory is independent of the velocity of the laboratory in space-time (Haugen and Lammerzahl, 2001; Pogosyan, 2011). In order to account for these findings in general relativity, Einstein in 1915 redefined several fundamental concepts (such as gravity and inertia) in terms of a new concept of curvature of space-time, instead of the more traditional system of forces understood by Newton (Einstein, 2001). Einstein proposed that there is no force of gravitation, but only the structure of space-time itself; gravity is the curvature of space-time caused by the local distribution of mass, momentum, and energy (Einstein, 2001; Tayler and Wheeler, 1966). General relativity is a geometric theory of gravitation (O'Connor and Robertson, 1996).

Fictitious forces are not necessary in general relativity, since its physics is explained with the geodesics of space-time (Ferraro, 2007).

Gravitational accelerations are strictly local and are the cumulative total effect of many local manifestations of curvature (Misner et al., 1973). In any region small enough for the curvature of space-time and other forces to be negligible, one can describe the region as an inertial frame (Einstein, 2001; Guilini, 2005); space-time is everywhere locally flat (Minkowski space-time), when considered on a sufficiently small scale, and an object follows a straight line in its local Lorentzian inertial frame (Chen, 2011; O'Neill, 1983; Tayler and Wheeler, 1966). If you let the space-time metric be curved, then gravity is suddenly explained. If the space-time 
metric possesses curvature, then the curvature will manifest as gravity (Einstein, 2001; Misner et al., 1973). In general relativity, the space-time metric $g$ describes the curvature of space-time; $g$ is related to and represents the gravitational field. The metric $g$ may be thought of as a device which makes corrections to the Minkowski metric for the description of gravity. Given any Lorentzian metric $g, g$ completely determines the gravitational field (Einstein, 2001; Misner et al., 1973).

In general relativity, the pertinent thing that typifies gravitation is the interaction between energy and momentum (mass times velocity) and the geometry of space-time; this is engendered by the Einstein Field Equations (EFE). The EFE are a set of ten nonlinear partial differential equations that describe the fundamental interaction of gravitation. First published by Einstein in 1915 as a tensor equation, the EFE are used to determine the space-time geometry resulting from the local distribution of mass-energy and linear momentum, i.e., they determine the metric tensor $g$ for a given arrangement of energy and momentum (Einstein, 2001). The EFE may be written in the simplified form: $E M=g-R$, where $E M$ is the energy-momentum (stress-energy) tensor, $g$ is the metric tensor, and $R$ is the Ricci curvature tensor. $g-R$ is called the Einstein tensor. Notice that the EFE have the same form as the Minkowski metric $\left(s^{2}=\Delta r^{2}-\right.$ $c^{2} \Delta t^{2}$ ); the EFE appear to be a modified form of the Minkowski metric to describe gravitation. $E M$ is a second-order tensor describing the local distribution of energy-momentum; it is the source of the gravitational field in general relativity (Einstein, 2001; Misner et al., 1973). EM has three components (Misner et al., 1973): (1) the electromagnetic energy-momentum tensor is a second-order tensor which describes the contribution to $E M$ due to the electromagnetic force field acting on matter, a light-like space-time interval interaction; (2) the pressure energymomentum tensor is a second-order tensor which describes the contribution to $E M$ due to the pressure force field acting on matter, a time-like space-time interval interaction; (3) the dissipation energy-momentum tensor is a second-order tensor which describes the contribution to $E M$ due to the dissipation force field acting on matter, a space-like space-time interval interaction. Any second-order tensor can be coupled to the energy-momentum tensor in the same way as gravity is (Misner et al., 1973). EM therefore consists of three space-time interval interactions which are the source of gravity in general relativity. $E M$ is analogous to $s^{2}$ of the Minkowski metric. $g$ is the second-order metric tensor (the metric) which computes the length of curves. In general relativity, the metric tensor $g$ is the fundamental object of study. The metric captures all the geometric and causal structure of space-time, being used to define notions such as time, distance, volume, curvature, angle, and separating the past and future (Misner et al., 1973). This is just what would be expected if $g$ was derived from the three basic types of space-time interval interactions. The metric (conventionally denoted by $g$ ) is given as a covariant second-order symmetric tensor on a four-dimensional differentiable curved manifold. The metric is nondegenerate with signature $(-+++)$. A manifold equipped with such a metric is a type of Lorentzian manifold (it supports light-like, time-like, and space-like intervals). The metric describes space-time curvature in a manner consistent with energy-momentum conservation. The $R$ (Ricci tensor) of the EFE is derived from $g$ and is the part of the curvature of space-time that determines the degree to which energy-momentum tends to converge or diverge in time (Chow and Knopf, 2004; Sidorov, 2001; Tahim et al., 2007). The Einstein tensor $g-R$ is a function of $g$ and expresses the curvature of space-time. The relationship between the metric tensor and the Einstein tensor allows the EFE to be written as a set of nonlinear partial differential equations. The solutions of the EFE are the components of the metric tensor. The inertial trajectories (geodesic paths) of particles and radiation are then 
calculated using the geodesic equations. The EFE reduce to Newton's Law of Gravitation when the gravitation field is weak and velocities are much less than the speed of light (Carroll, 2004).

The three basic types of space-time intervals are essentially the source of gravitation in general relativity; the general covariance of space-time intervals is extended to encompass gravitation. The general covariance of space-time intervals provides a link between observed physical reality (special relativity) and macroscopic physical reality (general relativity). When we observe large cosmic masses (moon, planets, stars, galaxies, etc.), at great distances from us, gravitational space-time curvature can be observed: gravitational lensing (luminous objects behind more massive objects become visible due to the bending of light around the more massive objects), gravitational redshift (light from objects moving away is shifted toward the red end of the electromagnetic spectrum), changes in the orbit of Mercury, frame-dragging (of space-time) around rotating bodies, and gravitational waves (ripples in the fabric of space-time). These and other predictions of general relativity have been confirmed by observations and experiments.

\section{Quantum Mechanics}

A goal of theoretical physicists working beyond the Standard Model is to quantize the gravitational field, yielding a quantum theory of gravity, which would unite gravity in a common theoretical framework with the other three fundamental interactions (electromagnetic, strong nuclear, weak nuclear). It is possible to describe gravity in the framework of quantum field theory like the other fundamental forces, such that gravity arises due to the exchange of virtual gravitons (Feynman et al., 1995; Zee, 2003). However, this fails at short distances of the order of the Planck length, where a more complete theory of quantum gravity (or a new approach to quantum mechanics) is required (Randall, 2005). If the space-time background of quantum mechanics is thought of as dynamic, then quantum gravity might be described, as in general relativity. Space and time would arise out of the relationships between events and space-time would have no existence apart from the events that occur within it; the only physically relevant information would be the relationships between events. Space-time intervals would then play a crucial role in the creation of the space-time and properties of quantum mechanics; space-time intervals could be the source of quantum gravity.

A quantum system is a portion of the physical world which is studied for its quantum mechanics. The condition that a quantum system is in at a particular time and place (point in space-time) is known as a quantum state (an event); a quantum state is the outcome of a measurement of a quantum system (Weinberg, 2002). Two quantum states (events) that occur are separated by a space-time interval. This space-time interval may be light-like, time-like, or space-like. If the space-time interval is light-like, then an electromagnetic interaction may occur between the two events. If a time-like space-time interval occurs between the two events, then a stress-pressure interaction may occur. If a space-like space-time interval occurs between the two events, a dissipation interaction may occur. In this way, space-time intervals create the space-time and properties of quantum mechanics; space-time intervals are the source of quantum gravity. Quantum gravity then, is not a force, but is the structure of space-time. Like in general relativity, quantum gravity unifies three fundamental interactions or forces (the three basic types of space-time intervals). The general covariance of space-time intervals is extended to quantum mechanics. Treating quantum gravity in this way seamlessly merges general relativity and quantum mechanics, providing a unified concept of gravity. Quantum gravity 
would reduce to general relativity in the classical limit and conform to Newton's law of gravitation in the weak-field limit (Zee, 2003; Feynman et al., 1995; Randall, 2005).

In physics, one often speaks of a field. Quantum field theories, such as the Standard Model, describe nature in terms of fields. General relativity is a field theory; at the core of general relativity are the Einstein Field Equations. In physics, a field is defined as a physical quantity that has a value for each point, pair of points, or other number of points in space and time (Gribbin, 1998; Feynman, 1970; McMullin, 2002). A space-time interval is a physical quantity that has a value for each pair of points in space and time; space-time intervals may be considered fields. In the modern parlance of quantum field theory, a field occupies space, contains energy and momentum (mass times velocity), and its presence precludes a classical true vacuum (Wheeler, 1998); fields have such familiar properties as energy content and momentum, just as particles can have (Feynman, 1970). The fact that a field can possess energy and momentum makes it very real. This has led physicists to consider fields as physical entities (particles, objects), making the field concept a supporting paradigm of modern physics. It was the introduction of the special theory of relativity which opened the way for physicists to start thinking of fields as truly independent entities (Weinberg, 1977). In quantum field theory, fields are understood as particles (electrons, protons, photons, etc.), elevating fields to the status of the most fundamental objects in the universe (Weinberg, 1977). Fields can be classified as scalar, vector, or tensor depending on whether the represented physical quantity is a scalar, vector, or tensor, respectively. Within each category (scalar, vector, tensor), a field can be either a classical field or a quantum field, depending on whether it is characterized by numbers or quantum operators.

Physicists have found that the notion of a field is of such practical utility in the analysis of force that a field is often considered to be a force (Feynman, 1970). In quantum field theory, a quantum field acts through the release of force carrier particles (messenger particles, intermediate particles) called "bosons" (Oerter, 2006). Each boson represents a quantum (a discrete quantity) of excitation of the relevant quantum field. There is one kind of field for every type of elementary particle (Weinberg, 1993). In quantum field theory, a force between two particles can be described either as the action of a field generated by one particle on the other, or in terms of the exchange of virtual bosons (quanta) between them. There are three conventionally accepted fundamental interactions or forces in quantum field theory (electromagnetic, strong nuclear, weak nuclear). These fundamental interactions (gauge interactions) are not reducible to more basic interactions; the three fundamental interactions result in all the forces we know (Schumm, 2004; Ulaby, 2010). The three, as part of the Standard Model of particle physics, are described as discrete quantum fields, and their interactions (excitations) are carried by bosons (quanta). The two nuclear interactions have short ranges, producing forces at minuscule, subatomic distances. The strong nuclear interaction, which is carried by the gluon, is responsible for the binding of quarks together to form hadrons (ordinary matter), such as protons and neutrons; as a residual effect, it binds the latter particles to form atomic nuclei (Ulaby, 2010). This is a stress-pressure interaction of the type carried by time-like space-time intervals. The weak nuclear interaction, which is carried by $\mathrm{W}$ and $\mathrm{Z}$ bosons, also acts on the nucleus, mediating radioactive decay (Ulaby, 2010). This is a dissipation interaction of the type carried by space-like space-time intervals. The electromagnetic fundamental interaction carried by the photon is significant at macroscopic scales where the effects can be seen directly in everyday life (Ulaby, 2010); this is of the type carried by light-like space-time intervals. There is a basic type of space-time interval that carries 
each quantum fundamental interaction. The three basic types of space-time intervals are consequently the source of quantum gravity; the general covariance of space-time intervals is extended to quantum mechanics.

Quantum mechanics has four phenomena which have not previously been explainable using classical physics (quantization, wave-particle duality, uncertainty, entanglement). These phenomena, however, are explainable utilizing space-time intervals, adding further support for the theory. The three basic types of space-time interval quantum fields act through the release of quanta (photons, gluons, and $\mathrm{W}$ and $\mathrm{Z}$ bosons). Light-like space-time intervals (the electromagnetic fundamental interaction) may underlie wave-particle duality. The waveparticle duality of the electromagnetic interaction has been observed many times (Brown, 2010; Thorne et al., 2004); wave and particle effects fully explain the emission and absorption spectra of electromagnetic radiation. Whether or not the electromagnetic interaction is described as a wave or particle depends on how a system is setup and observed. Space-like space-time intervals may explain quantum entanglement: the quantum states of two related particles cannot be described independently of one another and must be considered as a single system, no matter how far apart they may be (the two particles must have space-like separation for this to be realized; the particles could then be considered as a single system, distributed in space, but not in time). Time-like space-time intervals may explain quantum uncertainty (knowing about one property of a particle limits knowledge about other properties of the particle); signals or information can pass between particles with time-like space-time interval separation at less than the speed of light, causing interference and consequent uncertainty.

\section{Theory of Everything}

Finding a Theory of Everything (ToE) is one of the major unsolved problems of physics. A ToE is a hypothetical single all-encompassing coherent theoretical framework of physics that fully explains and links together all physical aspects of the universe (Weinberg, 1993). Over the past few centuries, two theoretical frameworks of physics have been developed that, as a whole, most closely resemble a ToE (Hawking, 2006). The two theories upon which all modern physics rests are general relativity (GR) and quantum field theory (QFT). GR is a theoretical framework that focuses on gravity for understanding the universe in regions of both large-scale and highmass. QFT is a theoretical framework that focuses on three fundamental forces for understanding the universe in regions of both small scale and low mass (Oerter, 2006). Since the usual domains of applicability of GR and QFT are so different, most situations require that only one of the two theories be used (Carlip, 2001; Jaeger, 2014; Priest, 2010). Physicists have experimentally confirmed with tremendous accuracy virtually every prediction made by these two theories when in their appropriate domains of applicability (Oerter, 2006). Scientists also have learned that GR and QFT, as they are currently formulated, are mutually incompatible-they cannot both be right. To resolve this conflict, a theoretical framework revealing a deeper underlying reality, unifying gravity with the three fundamental interactions (weak nuclear, strong nuclear, electromagnetic), must be discovered to harmoniously integrate the realms of GR and QFT into a seamless whole: a single theory that, in principle, is capable of describing all physical phenomena. Space-time intervals accomplish this. The general covariance of spacetime intervals underlies a reality which harmoniously integrates the realms of GR and QFT into a seamless whole, a single theory capable of describing all physical phenomena. Space-time intervals therefore underlie a single all-encompassing coherent theoretical framework of physics that fully explains and links together all physical aspects of the universe, i.e., a ToE. The hallmark of general covariance, the Minkowski metric, could serve as an axiom (premise, postulate) for this ToE. This ToE encompasses observed physical reality (conscious experience) 
as well; it fully explains and links together our observed physical reality and links it to physical reality. Space-time intervals are indeed the most fundamental objects in the universe.

\section{Dark Matter and Dark Energy}

The dark matter hypothesis plays a central role in current modeling of cosmic structure formation, galaxy formation and evolution, and on explanations of the anisotropies observed in the cosmic microwave background (the remnants of the big bang). All these lines of evidence suggest that galaxies, galaxy clusters, and the universe as a whole contain far more matter than that which is observable via electromagnetic signals (Siegfried, 1999). The standard model of cosmology indicates that the total mass-energy of the universe contains $4.9 \%$ ordinary matter, 26.8\% dark matter, and 68.3\% dark energy (NASA Mission Pages, 2013; NASA Science, 2015; Francis, 2013; Ade et al., 2013). Dark matter constitutes $84.5 \%$ of the total mass of the universe, while dark matter plus dark energy constitute $95.1 \%$ of the total mass-energy content (Carroll, 2007; Jarosik et al., 2011). No dark matter particle has ever been conclusively identified. Dark matter, however, could be a manifestation of space-time interval fields; space-time interval fields contain energy and momentum (mass times velocity) and may be considered particles or objects (matter). Dark matter has not been directly observed, but its existence and properties are inferred from its gravitational effects (Trimble, 1987). Space-time interval fields cannot be directly observed and are the source of gravitation. Space-time interval fields may be the hypothesized virtual particles, that pop in and out of existence, playing the role of dark matter (Wilkinson, 2017).

In physical cosmology and astronomy, dark energy is an unknown form of energy which is hypothesized to permeate all of space (Overbye, 2017; Peebles and Ratra, 2003). Dark energy is the most accepted hypothesis to explain the observations that the universe is expanding at an accelerating rate. Dark energy dominates the mass-energy of the universe; this may be because it is uniform across space (Steinhardt and Turok, 2006; Ferris, 2015). Space-time interval fields are uniform across space and occupy most of the universe. Dark energy is not very dense and is not known to interact through any of the fundamental forces other than gravity (Overbye, 2015). Space-time interval fields are unseen and not very dense; they are the source of gravity. Dark energy could be a manifestation of space-time interval fields. Dark energy would need to have a strong negative pressure (acting repulsively), like radiation pressure (Wang, 2016), to explain the observed acceleration of expansion of the universe. A dynamic field may be attractive or repulsive depending on the ratio of its kinetic and potential energy; the observed acceleration of expansion of the universe may be caused by the potential energy of a dynamical field (Ratra and Peebles, 1988; Caldwell, 2002). Potential energy is the energy possessed by an object because of its position, stresses within itself, its electric charge, and other factors (Jain, 2009; McCall, 2010). Space-time interval fields could have the high potential energy necessary for the acceleration of expansion of the universe.

\section{Conclusion}

Space-time intervals are the fundamental building blocks of nature. They may be the most fundamental objects in the universe. Space-time intervals are tiny bits of information (what, when, where) which interact to create space-time and it properties. Space-time intervals are the source of gravity in general relativity. Space-time intervals also underlie a quantum theory of gravity, which merges general relativity and quantum mechanics, providing a unified theory of gravity. Space-time intervals consequently underlie a single all-encompassing coherent theoretical framework of physics that fully explains and links together all physical 
aspects of the universe, a Theory of Everything. This Theory of Everything encompasses observed physical reality (conscious experience) as well; space-time intervals link observed physical reality to physical reality. Space-time intervals are devices by which we extract information from physical reality and utilize it to carry on our conscious lives. This information is accurate and reliable because of the link space-time intervals provide between observed physical reality and physical reality. The fact that human conscious experience (observed physical reality) is created through the processing of space-time intervals by the brain suggests that space-time intervals occupy a fundamental position in the universe and may be the fundamental basis of everything.

Space-time is dynamic. Space and time arise out of the relationships between events; space-time has no existence apart from the events that occur within it. The only physically relevant information are the relationships between events. Space-time intervals are relationships which give meaning and structure to space and time, creating space-time. The three basic types of space-time intervals are the three fundamental interactions or forces of nature. They cannot be reduced to more fundamental interactions or forces. All other known forces or interactions arise from space-time intervals. Space-time intervals are universal and ubiquitous in nature and because of their general covariance have a physical constancy. The general covariance of space-time intervals, as defined by the Minkowski metric, encompasses conscious experience, special relativity, general relativity, and quantum mechanics; the Minkowski metric is an axiom (premise, postulate) for a Theory of Everything.

It is interesting to observe how the general covariance of space-time intervals was developed and extended to encompass everything. The notion of general covariance was developed in special relativity to encompass inertial frames of reference. Special relativity was developed to explain observed physical reality, which is essentially conscious experience. There is an increasing amount of evidence suggesting that the brain processes the three basic types of space-time intervals to create human conscious experiences (Sieb, 2015, 2016, 2017a, 2017b); the general covariance of space-time intervals is extended to human conscious experience (observed physical reality). In general relativity, the three basic types of space-time intervals are the source of gravity; the general covariance of space-time intervals is extended to encompass gravitation. In quantum mechanics, the three basic types of space-time intervals are the three fundamental interactions or forces; the general covariance of space-time intervals is extended to encompass quantum gravity. Consequently, the general covariance of space-time intervals forms the basis of a Theory of Everything (it encompasses everything).

Space-time intervals are very important for human beings. They are the fundamental components of our conscious lives. Our entire daily activity is based on the processing of what, where, and when information carried by space-time intervals (Sieb, 2017a). We eat (what) at certain times (when) in certain places (where). We perform various tasks (what) at certain times (when) in certain places (where). Our entire conscious lives are directed and organized through the processing of what, where, and when information carried by space-time intervals. All of our higher cognitive processing and executive function (attention, perception, learning, memory, logic, planning, reasoning, calculating, decision-making, problem-solving, creative ability, intentional actions) is based on the what, where, and when information carried by space-time intervals. Scientific research at all levels is implemented through the collection and interpretation of the what, where, and when information carried by space-time intervals (Sieb, 2017a). It is important that scientists pay particular attention to space-time intervals in their collection and interpretation of data (Sieb, 2017a). Manipulation of space-time intervals may 
have important effects on results. There are a number of criteria based on space-time intervals which scientists might observe in conducting their research (Sieb, 2017a). Attention to spacetime intervals is also important in education, sports, physical therapy, medical diagnosis and treatment, treatment of psychiatric disorders (depression, psychosis, etc.), treatment of psychological disorders and problems (anxiety, etc.), and negotiating through our daily lives (these are discussed more thoroughly in Sieb, 2017a). Space-time intervals are indeed fundamental to our lives and activity.

Space-time intervals may be the fundamental basis of life. The processing of space-time intervals by the human brain creates conscious experience and this animates us, i.e., makes us come to life. A dead body no longer has consciousness and is no longer animated; it no longer processes space-time intervals. Space-time intervals may also animate plants and animals as well, giving them the appearance of being alive; all plants and animals do certain things (what), at certain times (when), in certain places (where). Perhaps it is space-time intervals then which give life to organisms. Space-time intervals have been largely ignored in scientific study, theory, and research. When vigorously applied, space-time intervals explain many of the mysteries of science (the nature of conscious experience, the subjectivity of conscious experience, the neural correlate of conscious experience, a unified concept of gravity, a Theory of Everything, the nature of dark matter and dark energy, the connection of our observed physical reality to actual physical reality, the nature of reality, the nature of life). Other mysteries and problems of science may also yield to the specter of space-time intervals (the origin of the Universe; the evolution of the Universe; the origin of life; more effective treatments for psychiatric, psychologic, and organic disorders; more effective scientific research; more effective learning and education; more effective physical training). Space-time intervals are the most fundamental objects in the universe.

\section{References}

Ade PAR, Aghanim N, Armitage-Caplan C, et al. Planck 2013 Results. l. Overview of products and scientific results-Table 9. Astronomy and Astrophysics 2013; 1303: 5062.

Baars B. A Cognitive Theory of Consciousness. Cambridge University Press, Cambridge, England, 1988.

Brown M. Physics for Engineering and Science ( ${ }^{\text {nd }}$ ed.). McGraw-Hill/Schaum, New York, 2013.

Burgess J and Rosen G. A Subject with No Object: Strategies for Nominalistic Reconstrual of Mathematics. Oxford University Press, New York, 1997.

Caldwell RR. A phantom menace? Cosmological consequences of a dark energy component with super-negative equation of state. Physics Letters B 2002; 545(1-2): 23-29.

Carlip S. Quantum gravity: A progress report. Reports on Progress in Physics 2001; 64(8): 885.

Carroll SM. Spacetime and Geometry: An Introduction to General Relativity. Addison-Wesley, San Francisco, 2004.

Carroll S. The Teaching Company, Dark Matter, Dark Energy: The Dark Side of the Universe. Guidebook Part 2 2007: 46. 
Chen B-Y. Pseudo-Riemannian Geometry with Applications to Relativity. World Scientific Publisher, Singapore, 2011.

Chow G and Knopf D. The Ricci Flow: An Introduction. American Mathematical Society, Providence RI, 2004.

Disalle R. Space and Time: Inertial Frames. The Stanford Encyclopedia of Philosophy (Winter 2009 Edition), E.N. Zalta (Ed.) 2009.

http://plato.stanford.edu/archives/sum2002/entries/spacetime-iframes/\#Oth

Einstein A. Relativity: The Special and the General Theory (Reprint of 1920 translation by Robert W. Lawson), Routledge, New York, 2001.

Ferris T. A First Glimpse of the Hidden Cosmos. National Geographic Magazine 2015 (January); 227(1): 108-123.

Ferraro R. Einstein's Space-Time: An Introduction to Special and General Relativity. Springer Science \& Business Media, 2007; 209-210.

Feynman RP. The Feynman Lectures on Physics Vol. II. Addison-Wesley Longman, San Francisco, 1970.

Feynman RP. Six Not-So-Easy Pieces: Einstein's Relativity, Symmetry, and Space-Time. Basic Books, New York, 1998.

Feynman RP, Morinigo FB, Wagner WG, Hatfield B. Feynman Lectures on Gravitation. Addison-Wesley, San Francisco, 1995.

Fields D. Galilean Relativity. Physics, University of New Mexico, Albuquerque, 2015; 262-301.

Francis M. First Planck Results: The Universe is still weird and interesting. Arstechnica (22 March 2013).

French AP. Special Relativity. MIT Introductory Physics Series. CRC Press, Florence, Kentucky, $1968 ; 86$.

Gribbin J. Q is for Quantum: Particle Physics from A to Z. Weidenfeld \& Nicolson, London, $1998 ; 138$.

Guilini D. Special Relativity. Cambridge University Press, Cambridge, England, 2005; 19.

Haugen MP and Lammerzahl C. Principles of Equivalence: Their Role in Gravitational Physics and Experiments that Test Them. Springer, New York, 2001.

Hawking SW. The Theory of Everything: The Origin and Fate of the Universe (Special Anniversary). Phoenix Books, Owen Sound, Ontario, 2006.

Hossenfelder S. Lost in thought. How important to physics was Einstein's imaginings? Scientific American 2015; 313(3). 
Jaeger G. What in the (quantum) world is macroscopic? American Journal of Physics 2014; 82(9); 896-905.

Jain MC. Fundamental Forces and Laws: a brief review. Textbook of Engineering Physics, Part 1. PHI Learning Pvt Ltd., Delhi, India, 2009: 10.

Jarosik N, et al. Seven-Year Wilson Microwave Anisotropy Probe (WMAP) Observations: Sky Maps, Systematic Errors, and Basic Results. Astrophysical Journal Supplement 2011; 192(2): 14.

Koch C. The neuroanatomy of visual consciousness. In: H.H.Jasper, L.Descarries,

V.F.Costelluchi, \& S.Rossignol (Eds.), Advances in Neurology, Consciousness at the Frontiers of Neuroscience. Lippencott-Raven, New York, 1998; 77: 229-239.

Landau LD and Lifshitz EM. Mechanics. Pergamon Press, New York, 196o; 4-6.

McCall RP. Energy, Work and Metabolism. Physics of the Human Body. John Hopkins University Press, Baltimore, Maryland, 2010: 74.

McMullin E. The Origins of the Field Concept in Physics. Physics in Perspective 2002; 4: 13-39.

Misner CW, Thorne KS, Wheeler JA. Gravitation. W.H. Freeman, San Francisco, 1973.

Morin D. Introduction to Classical Mechanics. Cambridge University Press, Cambridge, England, 2008.

Moskowitz C. Tangled up in spacetime. Scientific American (January) 2017.

Planck Mission Brings Universe into Sharp Focus. NASA Mission Pages. 21 March, 2013.

Dark Energy, Dark Matter. NASA Science: Astrophysics. 5 June 2015.

Oerter R. The Theory of Almost Everything: The Standard Model, the Unsung Triumph of Modern Physics. Plume, Kearny, NJ, 2006.

O’Connor JJ and Robertson EF. General Relativity. Mathematical Physics Index. School of Mathematics and Statistics. University of St. Andrews, Scotland, 2017.

O’Hanian HC and Ruffini R. Gravitation and Spacetime (2 ${ }^{\text {nd }}$ Ed.). W.W. Norton, New York, 1994 .

O’Neill B. Semi-Riemannian Geometry with Applications to Relativity, Pure and Applied Mathematics. Academic Press, New York, 1983; 103.

Overbye D. Astronomers Report Evidence of Dark Energy Splitting the Universe. The New York Times, 2015.

Overbye D. Cosmos Controversy: The Universe is Expanding, but How Fast? New York Times, 2017.

Peebles PJW and Ratra B. The cosmological constant and dark energy. Reviews of Modern Physics 2003; 75(2): 559-606. 
Penrose R. The Road to Reality. Vintage Books, London, 2005.

Petkov V. Minkowski Spacetime: A Hundred Years Later. Springer, Berlin, 2010.

Pogosyan P. Lecture 20: Black Holes-The Einstein Equivalence Principle. University of Alberta, Edmonton, 2011.

Priest SH. Encyclopedia of Science and Technology. Sage Publications, New York, 2010.

Purcell EM and Morin DJ. Electricity and Magnetism, (3 ${ }^{\text {rd }}$ Ed.). Cambridge University Press, Cambridge, England, 2013.

Randall L. Warped Passages: Unraveling the Universe's Hidden Dimensions. Ecco Press, New York, 2005.

Ratcliff JG. Foundations of Hyperbolic Manifolds. Springer-Verlag, New York, 2006.

Ratra B and Peebles PJE. Cosmological consequences of a rolling homogeneous scalar field. Physical Review D 1988; 37: 3406.

Roberts T and Schleif S. What is the experimental basis of special relativity? Usenet Physics FAQ, 2007.

Sartori L. Understanding Relativity: A simplified approach to Einstein's theories. University of California Press, Oakland, California, 1996; 9.

Schutz B. Gravity from the Ground Up: An Introduction to Gravity and General Relativity (Reprint Edition). Cambridge University Press, Cambridge, England, 2004.

Schumm BA. Deep Down Things: The Breathtaking Beauty of Particle Physics. John Hopkins University Press, Baltimore, Maryland, 2004.

Sidorov LA. Ricci Tensor. In: M.Hazewinkel (Ed.), Encyclopedia of Mathematics. Springer, New York, 2001.

Sieb RA. The emergence of consciousness. Medical Hypotheses 2004; 63(5): 900-904.

Sieb RA. Consciousness and voluntary action. In: SK Turrini (Ed.), Consciousness and Learning Research, Nova Science Publishers Inc., New York, 2007; 165-199.

Sieb RA. Consciousness and adaptive behavior. Activitas Nervosa Superior 2011; 53(N.1-2), 2126.

Sieb RA. The emergence of emotion. Activitas Nervosa Superior 2013; 55(4): 115-145.

Sieb RA. Memory in four dimensions. In: M.Sakakibara \& I.Etsuro (Eds.), Memory Consolidation, Chapter 13, 259-313. Nova Science Publishers, Inc., New York, 2015.

Sieb RA. Human conscious experience is four-dimensional and has a neural correlate modeled by Einstein's special theory of relativity. Neuroquantology 2016; 14(4): 630-644. 
Sieb RA. The what, where, and when of consciousness and psychology research. Advances in Psychology Research, AM Columbus (Ed.), Nova Science Publishers Inc., New York, 2017a; 122: 19-57.

Sieb RA. Four-Dimensional Consciousness. Activitas Nervosa Superior 2017b; 59: 43-6o.

Siegfried T. Hidden Space Dimensions May Permit Parallel Universes, Explain Cosmic Mysteries. The Dallas Morning News, 1999.

Smolin E. Three Roads to Quantum Gravity. Basic Books, New York, 2001; 2025.

Steinhardt PJ and Turok N. Why the cosmological constant is small and positive? Science 2006; 312(5777): 1180-1183.

Tahim MO, Landim RR, Almeida CAS. Spacetime as a deformable solid. General Relativity and Quantum Cosmology. Cornell University Library, Ithaca, New York, 2007.

Tayler EF and Wheeler JA. Spacetime Physics: Introduction to Special Relativity ( ${ }^{\text {st }}$ ed.). Freeman, San Francisco, 1966; 175-190.

Thorn JJ, Neel MS, Donato VW, Bergreen GS, Davies RE, Beck M. Observing the quantum behavior of light in an undergraduate laboratory. American Journal of Physics 2004; 72(9): 1210.

Tononi G and Edelman G. Consciousness and the integration of information in the brain. In: H.H.Jasper, L.Descarries, V.F.Costelluchi, \& S.Rossignol (Eds.), Advances in Neurology, Consciousness at the Frontiers of Neuroscience. Lippencott-Raven, New York 1998; 77: 245280.

Trimble V. Existence and nature of dark matter in the universe. Annual Review of Astronomy and Astrophysics 1987; 25: 425-472.

Ulaby FT, Michielssen E, Ravaioli R. Fundamentals of Applied Electromagnetics (6 $6^{\text {th }}$ ed.). Prentice Hall, Boston, 2010.

Wang Z-Y. Modern Theory for Electromagnetic Metamaterials. Plasmonics 2016; 11(2): 503508.

Weinberg S. The Search for Unity: Notes for a History of Quantum Field Theory. Daedalus 1977; 106(4): 17-35.

Weinberg S. Dreams of a Final Theory: The Scientists Search for the Ultimate Laws of Nature. Knopt Doubleday Publishing Group, New York, 1993.

Weinberg S. The Quantum Theory of Fields, 1, Cambridge University Press, Cambridge, England, 2002.

Weinberg S. Physics: What We Do and Don’t Know. New York Review of Books, 2013.

Wessen PS. Five-Dimensional Physics. World Scientific, Singapore, 2006; 82.

Wheeler J and Ford K. Geons. Black Holes, \& Quantum Foam: A Life in Physics. W.W. Norton, New York, 1998; 163.

Wilkinson G. Measuring beauty. Scientific American 2017; 317(5): 57-63. 
Zee A. Quantum Field Theory in a Nutshell. Princeton University Press, Princeton, New Jersey, 2003. 